\title{
Lévitation électromagnétique de gouttes - présentation du projet MAGLEV - premiers résultats
}

\author{
Jacqueline Etay ${ }^{\mathrm{a}}$, Yves Fautrelle, Annie Gagnoud, Yves Duterrail, \\ DAMien Perrier et Benô̂t Bardet \\ CNRS et INPG, EPM-Madylam, ENSHMG BP95 38402 St Martin d'Hères Cedex, France
}

Reçu le 8 octobre 2003, accepté le 19 janvier 2004

\begin{abstract}
Résumé - Il est admis qu'une bonne connaissance des propriétés thermophysiques d'alliages métalliques à l'état liquide est déterminante pour la maîtrise de leur élaboration. Ces propriétés peuvent être mesurées, entre autres, au moyen d'un dispositif de lévitation électromagnétique. Des expériences de ce type ont été menées à bien dans les années passées en micro pesanteur dans un projet ESA, intitulé TEMPUSContainerless Processing in Space. Des résultats originaux ont été obtenus. Néanmoins, la présence de mouvements de brassage électromagnétique dans la goutte fondue engendre de nombreux problèmes d'instabilités et une précision dégradée des mesures réalisées. L'étude des mouvements fait l'objet d'un programme intitulé «MAGLEV » dont le volet français est soutenu par le CNES. La contribution de notre équipe présente deux volets. L'un concerne l'effet de la modulation des courants inducteurs sur les oscillations de la surface libre et les possibilités de contrôle qu'offre cette modulation. L'autre volet est centré sur l'électromagnétisme du système pour lequel des exemples de calculs sont présentés Nous présentons d'abord notre installation expérimentale et le dispositif original de diagnostic et de pilotage qui est mis en œuvre sur un modèle. Une simulation numérique de l'installation expérimentale suit. Les perspectives sont développées.
\end{abstract}

Mots clés : Lévitation électromagnétique / induction / courants électriques bi-fréquence

Abstract - Electromagnetic levitation of dooplet - MAGLEV project presentation - first results. A good knowledge of the thermo physical properties of liquid metal alloys is of major importance for processing. For this purpose, an electromagnetic levitator may be used. This has been performed in the past by an ESA project called TEMPUS-Containerless Processing in Space. Results were obtained. Nevertheless, stirring motions in the molten drop generate instability problems, and lead to a deteriorated accuracy of the measure. The stirring motions will be studied in the framework of a future ESA project called "MAGLEV". The national french agency CNES is supporting the french part. Our participation is twofold. First, we look at the effect of the modulation of inducting currents on the oscillations of the free surface. The experimental set-up is presented as well as its original device devoted to the driving of the experiment and the diagnostic on a test experiment. Second, a numerical simulation of the device is done. Perspectives of modelling are developed.

Key words: Electromagnetic levitation / induction / electrical two-frequency currents

\section{Introduction}

La propriété de lévitation des champs magnétiques alternatifs est largement utilisée pour mesurer les propriétés physiques d'alliage métalliques : masse volumique, tension superficielle, viscosité, résistivité, capacité calorifique, conductivité thermique. Le tableau 1 donne un résumé de la façon dont les propriétés physiques sont mesurées, notamment dans un contexte spatial.

\footnotetext{
a Auteur correspondant : etay@grenoble.cnrs.fr
}

Les phénomènes physiques sur lesquels ce type de dispositif s'appuie peuvent être résumés de la façon suivante.

Un milieu de conductivité électrique $\sigma$, de volume $V$, placé dans un champ magnétique $\mathbf{B}$ d'intensité caractéristique $B_{0}$ alternatif de pulsation $\omega_{1}$, développe des courants électriques induits de densité $\mathbf{j}$. Ce phénomène a deux effets sur ce milieu appelé « charge » : un effet thermique et un effet mécanique.

L'effet thermique est aussi appelé « chauffage par effet Joule ». Il est utilisé pour fondre la charge et la maintenir en température. 
Tableau 1. Récapitulatif des principes des mesures de propriétés thermophysiques mesurées par les dispositifs de lévitation électromagnétique.

\begin{tabular}{|c|c|c|c|}
\hline propriétés & principe de la mesure & mesure de ou des ... & appareillage utilisé \\
\hline masse volumique & $\begin{array}{l}\text { sur terre : équilibre des } \\
\text { forces de gravité et des } \\
\text { forces électromagnétiques } \\
\text { en microgravité } \\
\text { équilibre des forces d'inertie } \\
\text { et de tension superficielle }\end{array}$ & position de la charge & \\
\hline $\begin{array}{l}\text { tension } \\
\text { superficielle }\end{array}$ & $\begin{array}{l}\text { mise en oscillation de la } \\
\text { goutte fluide par modulation } \\
\text { du courant inducteur }\end{array}$ & $\begin{array}{l}\text { fréquences excitées à la } \\
\text { surface libre de la charge }\end{array}$ & $\begin{array}{l}\text { caméra haute résolution } \\
\text { de } 30 \text { à } 300 \text { images par } \\
\text { seconde }\end{array}$ \\
\hline viscosité & $\begin{array}{l}\text { impulsion de courant } \\
\text { inducteur }\end{array}$ & $\begin{array}{l}\text { temps d'amortissement } \\
\text { des oscillations }\end{array}$ & \\
\hline résistivité & $\begin{array}{l}\text { mesure de la puissance } \\
\text { Joule dissipée dans la } \\
\text { charge }\end{array}$ & $\begin{array}{l}\text { ddp et fréquence dans le } \\
\text { circuit d'alimentation de } \\
\text { l'inducteur }\end{array}$ & $\begin{array}{l}\text { voltmètre et } \\
\text { fréquencemètre }\end{array}$ \\
\hline $\begin{array}{l}\text { capacité } \\
\text { calorifique }\end{array}$ & $\begin{array}{l}\text { modulation du courant } \\
\text { inducteur }\end{array}$ & $\begin{array}{l}\text { mesure temporelle de la } \\
\text { température de surface } \\
\text { de la goutte }\end{array}$ & pyromètre \\
\hline $\begin{array}{l}\text { conductivité } \\
\text { thermique }\end{array}$ & $\begin{array}{l}\text { impulsion du courant } \\
\text { inducteur }\end{array}$ & $\begin{array}{l}\text { déphasage entre la } \\
\text { modulation de la } \\
\text { la température de surface } \\
\text { et la modulation de la } \\
\text { consigne d'alimentation } \\
\text { du courant l'inducteur }\end{array}$ & $\begin{array}{l}\text { fréquencemètre et } \\
\text { pyromètre }\end{array}$ \\
\hline
\end{tabular}

L'effet mécanique est dû aux forces électromagnétiques $\mathbf{F}$ :

$$
\mathbf{F}=\mathbf{j} \times \mathbf{B}
$$

qui présentent une partie moyenne $\langle\mathbf{F}\rangle$ et une partie pulsante $\mathbf{F}_{\mathbf{p}}$ et $\mathbf{F}=\langle\mathbf{F}\rangle+\mathbf{F}_{\mathbf{p}}$. Le rapport $|\langle\mathbf{F}\rangle| /\left|\mathbf{F}_{\mathbf{p}}\right|$ augmente comme la racine carrée du paramètre d'écran $R_{\omega}$, mesure de l'épaisseur de diffusion du champ magnétique à l'intérieur de la charge (l'épaisseur de peau électromagnétique) comparée à la dimension caractéristique de la charge $a$ :

$$
R_{\omega}=\mu \sigma \omega_{1} a^{2}
$$

où $\mu=4 \pi \cdot 10^{-7}$ SI est la perméabilité magnétique du vide, $a$ la dimension caractéristique de la charge définie par $a=(3 V / 4 \pi)^{1 / 3}$.

Ainsi, pour une fréquence $f_{1}=\omega_{1} / 2 \pi$ et une intensité de champ magnétique suffisamment élevées, le milieu peut être mis en lévitation. Les forces électromagnétiques équilibrent alors les forces de gravité (Okress et al. [1]), c'est-à-dire :

$$
\frac{B_{0}^{2}}{2 \mu \rho g a} \approx O(1)
$$

où $g$ est la gravité, $\rho$ la masse volumique du milieu considéré.

Outre la lévitation, les forces électromagnétiques engendrent du brassage électromagnétique. La vitesse caractéristique $U$ de ce brassage est proportionnelle à la vitesse d'Alfvén $U_{\mathrm{A}}=B_{0} / \sqrt{\mu \rho}$.

$$
U=\alpha \frac{B_{0}}{\sqrt{\mu \rho}}
$$

avec $\alpha$ un paramètre qui dépend de la géométrie utilisée.

Enfin, les fréquences des oscillations de la charge qui apparaissent sur ce type de dispositif sont (voir par exemple Cummings [2])

- d'une part une oscillation verticale globale, dûe au fait que, sur terre, le centre de gravité de la charge en lévitation ne correspond pas au point de champ magnétique nul. On remarque que, dans l'espace, cette source d'instabilité potentielle est fortement réduite voire supprimée. La fréquence de cette oscillation est notée $f_{v}$;

- d'autre part, les oscillations naturelles de la surface libre, que l'on appelle souvent les oscillations de Rayleigh (Lamb [3]). C'est la première de ces instabilités, le mode d'oscillation 2, que l'on excite lors des mesures de tension superficielle et de viscosité dans l'expérience Tempus-MSL. Sa fréquence est notée $f_{2}$.

Ces 2 types d'oscillations sont schématisés sur la figure 1

$$
f_{v}=\frac{1}{2 \pi} \sqrt{\frac{g}{2 \Delta z_{0}}}
$$

où $\Delta z_{0}$ est la différence de cote entre la position verticale du centre de gravité de la charge en lévitation et la position du point de champ nul. Lorsque $\Delta z_{0}$ tend vers zéro, la fréquence $f_{v}$ augmente et l'amplitude des oscillations associées tend vers zéro

$$
f_{2}=\frac{1}{2 \pi} \sqrt{\frac{8 \gamma}{\rho a^{3}}}
$$

où $\gamma$ est la tension superficielle de la charge fondue que l'on appelle aussi goutte. 

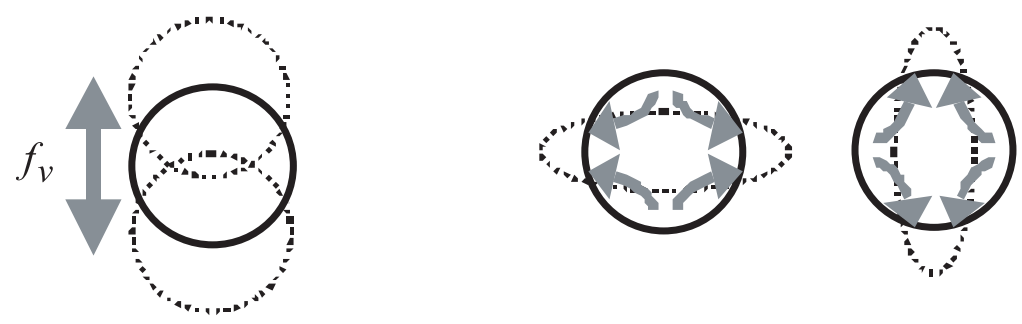

Fig. 1. Schéma illustrant les 2 types d'oscillation de la charge en lévitation. À gauche oscillation globale verticale, à droite oscillation naturelle mode 2.

\section{Contexte spatial - L'expérience TEMPUS-MSL}

Pour obtenir les propriétés physiques d'alliages surfondus, et afin de réduire l'intensité du brassage électromagnétique et les sources d'instabilité, l'expérience TEMPUS-MSL, pour MagnetoStatic Levitator, a été mise en œuvre. Des expériences ont été menées à bien dans les années passées en micro-pesanteur pour lesquelles un dispositif électromagnétique (Fig. 2) comportant deux inducteurs a servi d'une part à centrer une charge d'alliage métallique de diamètre $1 \mathrm{~cm}$ environ, d'autre part à la fondre et à la maintenir en température et le cas échéant à exciter des oscillations de sa surface. Vidéo, pyromètres, et système de mesure laser viennent compléter ce dispositif.

Des résultats originaux, d'une grande portée industrielle, ont été obtenus (Egry [4]). Toutefois, ces expériences présentent deux défauts. D'une part, il arrive qu'elles doivent être interrompues, la charge s'étant déstabilisée. D'autre part, les mesures ne sont pas toujours reproductibles car les effets du brassage électromagnétique sur les mesures ne sont pas correctement décrits. Enfin l'interprétation des signaux mesurés, grâce à une source laser, du mouvement du point supérieur de la goutte, nécessite a posteriori un traitement adapté du signal pour supprimer les parasites dus aux vibrations annexes.

Pour apporter des réponses à ces 2 problèmes, un consortium européen appelé MAGLEV s'est mis en place. La contribution française à ce travail présente 2 volets, un volet expérimental et un volet numérique. La partie expérimentale doit servir :

- à valider l'utilisation d'un dispositif de mesure de tension superficielle original, dont le principe et des résultats obtenus dans une configuration test de creuset sont présentés dans la section 3.2 de ce papier;

- à valider et tester le caractère dimensionnant de nos codes de calcul présentés dans la section 4 .

L'installation expérimentale que nous avons mise en œuvre est présentée dans la section 3.1 ainsi que les ordres de grandeur qui lui sont attachés. La méthode proposée pour le diagnostic d'état d'agitation de la charge est ensuite présentée (Sect. 3.2) ainsi que des résultats obtenus sur une configuration plus simple à mettre en œuvre que celle correspondant à la lévitation. Les résultats expérimentaux et de simulation numérique que nous

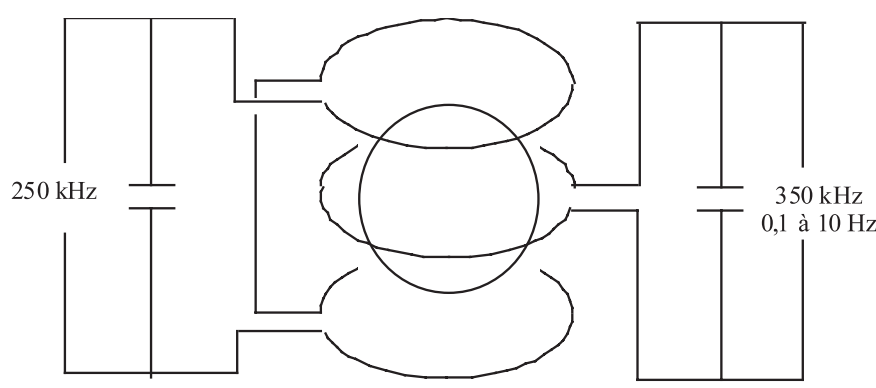

lévitation - centrage

chauffage - excitation

Fig. 2. Schéma électrique du dispositif TEMPUS-MSL. Un inducteur centre la charge et l'autre inducteur la chauffe et l'excite mécaniquement.

avons obtenus relatifs à l'expérience suivent (Sect. 4.1). Des prospectives d'utilisation de code sont présentées dans la section 4.2. Ils permettront à terme de décrire avec plus de précision non seulement les expériences de lévitation mais aussi tous les dispositifs d'induction.

\section{Le dispositif expérimental Maglev}

\subsection{La cellule de mesure}

Une expérience de lévitation électromagnétique a été montée au laboratoire. Elle comporte 3 parties : une cellule d'expérience, une partie électrotechnique, une partie acquisition. La cellule (Fig. 3 à gauche) est une enceinte constituée d'un tube de quartz de diamètre $140 \mathrm{~mm}$ et fermée par 2 flasques. La flasque du bas, en matière isolante électriquement, autorise à la fois le passage des arrivées de courant dans l'inducteur et celui d'une tige de support mobile (Fig. 3 au centre) permettant la mise en place de la charge solide dans l'inducteur. La flasque du haut, en acier, permet $(i)$ de faire le vide, un coude permettant le raccordement à une pompe à vide, (ii) de mettre l'enceinte sous argon, (iii) le positionnement éventuel d'une sonde de contact ou d'un thermocouple. La partie électrotechnique est constituée d'un générateur à triodes Celes alimentant un circuit oscillant constitué d'un coffret de capacités et d'un inducteur (Fig. 3 au centre). La géométrie de l'inducteur a été choisie identique à l'expérience numérique de Bojarevitch [5]. Il est composé de 4 spires et de 2 contre-spires. La partie contrôle et acquisition de l'expérience est présentée en fin de section 4 . 

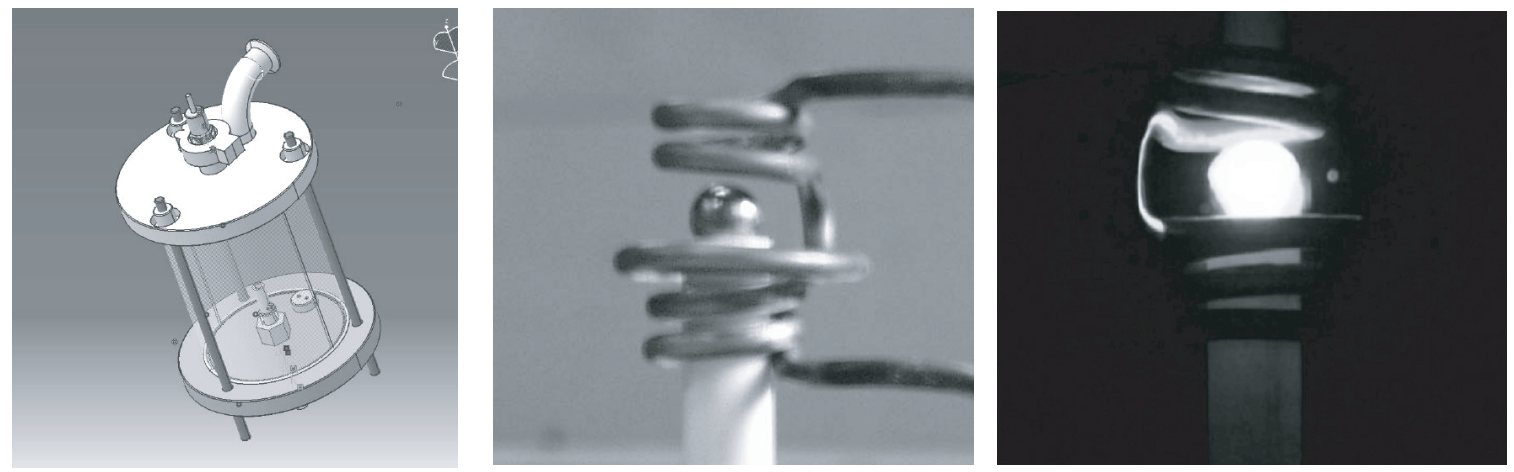

Fig. 3. Expérience Maglev, à gauche l'enceinte de test, au centre la sphère de nickel sur son support avant fusion, à droite la charge de nickel fondue et en lévitation.

Tableau 2. Données thermophysiques du nickel (Smithells [6]).

\begin{tabular}{lc}
\hline température de fusion & $T_{f}=1454{ }^{\circ} \mathrm{C}$ \\
masse volumique (à Tf) & $\rho=7905 \mathrm{~kg} \cdot \mathrm{m}^{-3}$ \\
tension de surface du nickel (à Tf) & $\gamma=1,778 \mathrm{~N} \cdot \mathrm{m}^{-1}$ \\
capacité calorifique du nickel (à Tf) & $c_{\mathrm{p}}=444 \mathrm{~J} \cdot \mathrm{kg}^{-1} \cdot \mathrm{K}^{-1}$ \\
chaleur latente du nickel & $\Delta H=292 \times 10^{3} \mathrm{~J} \cdot \mathrm{kg}^{-1}$ \\
viscosité du nickel liquide (à Tf) & $\rho \nu=0,0049 \mathrm{~kg} \cdot \mathrm{m}^{-1} \cdot \mathrm{s}^{-1}$ \\
\hline
\end{tabular}

Tableau 3. Ordres de grandeur de l'expérience Maglev.

\begin{tabular}{lcc}
\hline diamètre caractéristique de la charge $: 2 a$ & & $10 \mathrm{~mm}$ \\
fréquence de base du courant inducteur : $f_{1}$ & $303 \mathrm{kHz}$ \\
paramètre d'écran : $R_{\omega}=\mu \sigma \omega_{1} a^{2}$ & (Éq. (2)) & 82 \\
champ magnétique nécessaire à la lévitation : $B_{0} \sim \sqrt{2 \mu \rho g a}$ & (Éq. (3)) & 317 Gauss \\
vitesse d'Alfvén : $U_{\mathrm{A}}=B_{0} / \sqrt{\mu \rho}$ & (Éq. (4)) & $0,32 \mathrm{~m} . \mathrm{s}^{-1}$ \\
nombre de Reynolds $R_{\mathrm{A}}=\frac{U_{\mathrm{A}} a}{\nu}$ & & 2500 \\
fréquence d'oscillation mode $2: f_{2}=\frac{1}{2 \pi} \sqrt{\frac{8 \gamma}{\rho a^{3}}}$ & (Éq. (6)) & $19,1 \mathrm{~Hz}$ \\
fréquence d'oscillation verticale $f_{v}=\frac{1}{2 \pi} \sqrt{\frac{g}{2 z_{0}}}$ & (Éq. (5)) & $11,15 \mathrm{~Hz}$ \\
& & pour $\Delta z_{0}=1 \mathrm{~mm}$ \\
\hline
\end{tabular}

La charge a été choisie comme du nickel dont les propriétés thermophysiques sont listées dans le tableau 2. Les ordres de grandeur liés à l'expérience sont portés dans le tableau 3.

\subsection{Le détecteur de résonance - Effet d'un champ magnétique bi-fréquence sur la dynamique d'une surface libre}

Nous souhaitons développer un système capable d'exciter les oscillations naturelles de la goutte en lévitation et de les détecter sans contact matériel. Pour cela, nous souhaitons moduler le courant dans l'inducteur à ses fréquences de résonance afin de produire les oscillations présentant une amplitude maximale.
Pour cela, nous modulons donc le courant inducteur $I$ à une fréquence variable $f_{2}=\omega_{2} / 2 \pi$, telle que $f_{2} \ll f_{1}$ c'est-à-dire $I=I_{0}\left(1+\alpha \cos \omega_{2} t\right) \cos \omega_{1} t$ où $\alpha<1$ est le coefficient de modulation. Dans ce cas, il a été montré (Perrier et al. [7]) que les forces électromagnétiques dans la charge se mettent sous la forme :

$$
\langle\mathbf{F}\rangle=\left\langle\mathbf{F}_{0}\right\rangle\left\{\left(1+\frac{\alpha^{2}}{2}\right)+\tilde{m}(t)\right\}
$$

où $\left\langle\mathbf{F}_{0}\right\rangle$ est la force électromagnétique pour $\alpha=0$ et $\tilde{m}(t)=\frac{\alpha}{2} \cos 2 \omega_{2} t+2 \alpha \cos \omega_{2} t$.

La surface libre d'un domaine liquide soumis à de telles forces se comporte comme un oscillateur forcé à la 


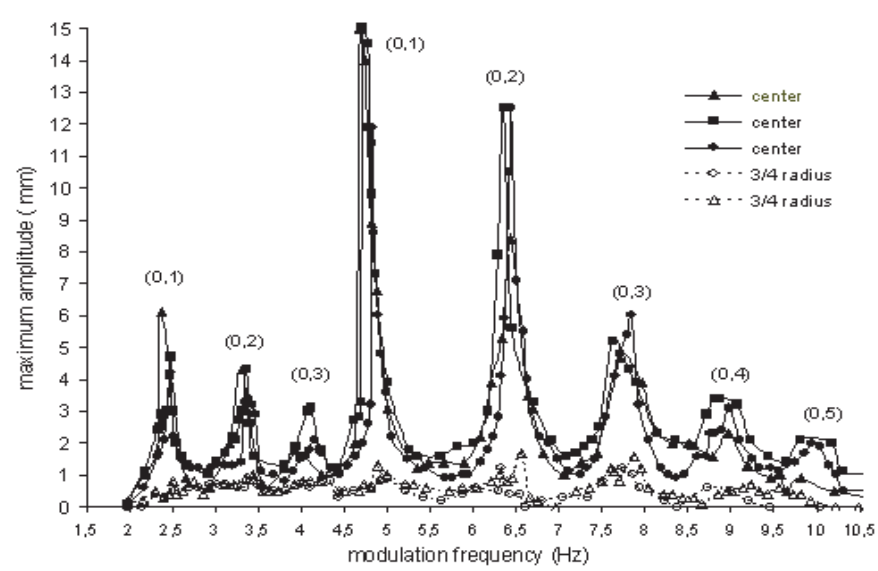

Fig. 4. Amplitude maximale des déformations de la surface libre pour différentes fréquences de modulation $f_{2}$. Les modes de surface $(0, n)$ sont notés au-dessus des pics de résonance correspondants. Mesures réalisées dans la configuration gallium + creuset.

fréquence $f_{2}$ ou $2 f_{2}$. C'est-à-dire que sa surface obéit à l'évolution spatio-temporelle $\eta(\mathbf{x}, t)$ suivante :

$$
\begin{aligned}
\eta(\mathbf{x}, t)=\eta_{0}(\mathbf{x})+\sum_{n=1}^{\infty} \frac{A_{n}}{\left(\Omega_{n}^{2}-\omega_{2}^{2}\right)} E_{n}(\mathbf{x}) \cos \omega_{2} t \\
\quad+\sum_{n=1}^{\infty} \frac{B_{n}}{\left(\Omega_{n}^{2}-4 \omega_{2}^{2}\right)} E_{n}(\mathbf{x}) \cos 2 \omega_{2} t
\end{aligned}
$$

où $\eta_{0}(\mathbf{x})$ est la position de la surface libre en absence de modulation, $E_{n}(\mathbf{x})$ sont les fonctions d'une base orthogonale, et $\Omega_{n}$ les fréquences propres des oscillations de type Rayleigh. Le fait que $\eta_{0}(\mathbf{x})$ ne soit pas une sphère est appelé le formage électromagnétique : les parties de la surface libre les plus proches des inducteurs sont repoussées de ces régions, tandis que le liquide tend à occuper les régions de champ magnétique plus faible. C'est cette forme qui sera, pour une configuration axisymétrique, calculée en section 4.1 de ce papier.

Sur un dispositif dans lequel la charge est un bain de gallium $\left(a_{\mathrm{Ga}}=42 \mathrm{~mm}\right.$ et $\left.h_{\mathrm{Ga}}=45 \mathrm{~mm}\right)$ contenu dans un creuset pyrex et entouré par un inducteur solénoïdal, il a été montré (Perrier et al. [7]) que ce type de forces était capable de forcer différents modes de surface. Une pointe de contact a permis de caractériser les déformations associées à ces modes pour différentes fréquences de modulation $f_{2}$. Les résultats de ces mesures sont portés sur la figure 4. Des amplitudes d'oscillations du même ordre de grandeur que $a_{\mathrm{Ga}}$ ont été observées.

Parallèlement à ce système de mesure avec contact, un dispositif original de détection sans contact du comportement dynamique de la charge a été testé. Son principe est basé sur le fait que le circuit électrique oscillant, formé d'un coffret de capacités $C$ d'une part et du système « inducteur + charge » d'inductance $L$ d'autre part, oscille à la résonance : $L C \omega_{1}^{2}=1$. Ainsi, puisque $C$ est fixe, une mesure temporelle de la fréquence de base $f_{1}$ donne la variation de $L$. La figure 5 représente

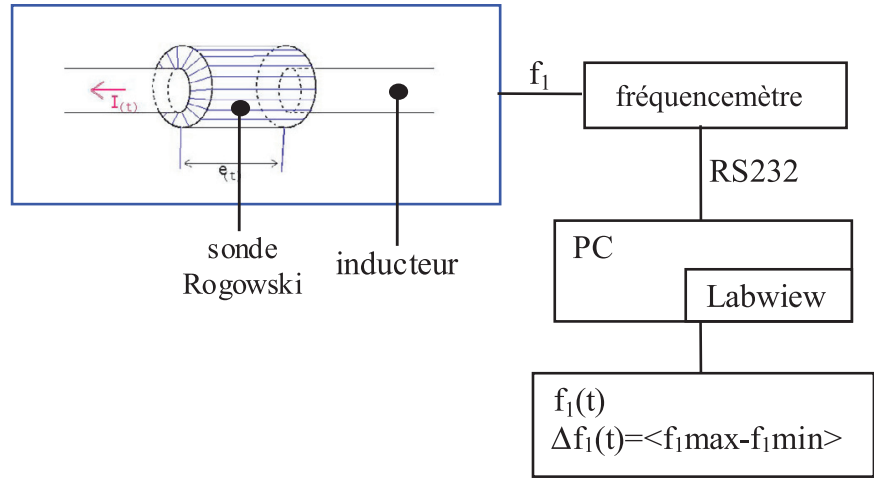

Fig. 5. Schéma de l'acquisition et du traitement de la fréquence.

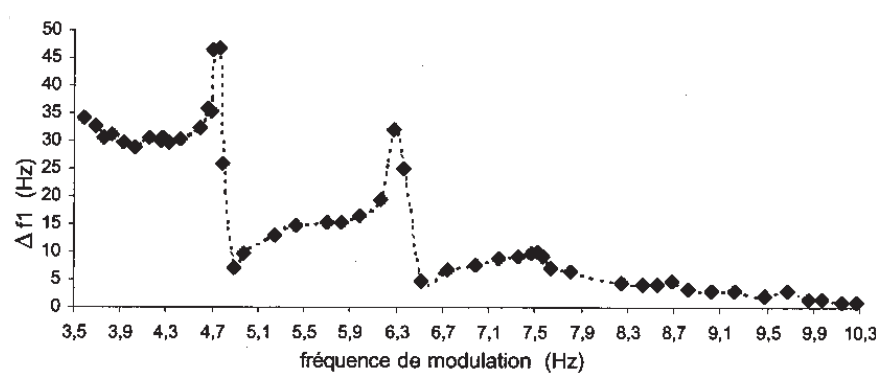

Fig. 6. Variation de $\Delta f_{1}=f_{1}\left(f_{2}\right)-f_{1}(0)$ en fonction de la fréquence de modulation. On observe les mêmes pics de résonance que ceux de la figure 4, Mesures réalisées dans la configuration gallium + creuset.

la chaîne d'acquisition et de traitement du signal. Plus de précision sur le principe de cette mesure peut être trouvée dans Perrier [8]. Les variations mesurées de la différence $\Delta f_{1}=f_{1}\left(f_{2}\right)-f_{1}(0)$ en fonction de la fréquence de modulation $f_{2}$ sont portées sur la figure 6 . Sur cette figure, les pics correspondent aux mêmes valeurs de $f_{2}$ que les pics de la figure 4. Ce dispositif est donc prometteur non seulement pour mieux connaître la dynamique de la charge dans l'expérience Maglev, mais aussi pour tous les procédés inductifs. Afin de contrôler la dynamique des surfaces libres nous envisageons d'asservir la valeur de la fréquence $f_{2}$ à la mesure de $\Delta f_{1}$ afin de piloter l'excitation automatique de la première résonance.

\section{Simulation numérique et mise en place des futures modélisations numériques}

\subsection{Simulation numérique de l'expérience Maglev}

Afin de simuler le comportement hydrodynamique de la goutte en lévitation dans notre l'expérience présentée à la section 3 , en régime stationnaire, et en géométrie axisymétrique, nous avons couplé, par fichiers, 2 logiciels : Ophélie, un code maison (Gagnoud [9]) et le logiciel commercial Fluent muni d'un module de calcul de forces électromagnétiques utilisant la loi de Biot-Savart « Induct $2 \mathrm{D} »($ Delannoy $[10])$. 
Le logiciel Ophélie est un logiciel de calcul d'électromagnétisme basé sur une méthode d'intégrales de frontière. Pour une tension donnée aux bornes de l'inducteur, il calcule la densité des courants dans cet inducteur, la densité des courants induits dans la charge, les puissances et forces associées à ces courants induits ainsi que toutes les valeurs électromagnétiques associées. DE plus, une minimisation de l'énergie totale du système (énergie électromagnétique + énergie de surface + énergie potentielle) permet le calcul de la forme $\eta_{0}(\mathbf{x})$ de la goutte en lévitation. On note que dans ces calculs, les courants inducteurs et induits sont ramenés à la surface du milieu dans lesquels ils circulent, leur intensité étant considérée en décroissance exponentielle dans la peau électromagnétique. Comme dans le cas expérimental, l'épaisseur de peau électromagnétique est égale à $16 \%$ du rayon de la charge, cette approximation, propre aux éléments de frontière, engendre une erreur de $20 \%$ environ sur la puissance Joule dissipée dans la charge.

Le module Induct-2D permet le calcul, pour une distribution donnée de courants inducteurs, de la densité des courants induits dans la charge et des grandeurs électromagnétiques associées. Les forces de volume ainsi calculées sont prises en compte dans l'équation de NavierStokes permettant le calcul de l'écoulement. Rien n'est prévu dans ce code ni pour calculer une déformation de la surface libre, ni pour positionner la charge en position d'équilibre.

Afin de bénéficier des fonctionnalités de chacun de ces codes et de les utiliser au mieux, nous avons opté pour la stratégie suivante. Grâce à Ophélie, pour une intensité totale donnée dans chacune des spires inductives, nous calculons la forme et la position de la goutte. Cette forme et cette position sont données comme la géométrie d'entrée de Fluent. Là, grâce à Induct 2D, les forces sont à nouveau calculées, sans l'approximation MIF, et le centre de gravité de la charge est réajusté pour obtenir l'équilibre entre l'intégrale des forces électromagnétiques sur le volume et le poids de la charge. Le calcul de l'écoulement est alors réalisé.

Le résultat du calcul Ophélie est porté sur la figure 7. On y voit la géométrie de l'inducteur qui correspond à celle de la figure 3 . Les propriétés thermophysiques injectées dans le logiciel sont celles du tableau 2. L'intensité du courant inducteur est de 400 ampères efficaces et la fréquence de $303 \mathrm{kHz}$. Ce courant est double de celui qui serait nécessaire pour la simple production du champ magnétique issue de l'équation (3). Sur la partie gauche, le domaine est sphérique, sur la partie droite, la surface du domaine est déformée. Leur position respective correspond à l'équilibre. Pour le courant choisi, le surface n'est pas tres éloignée de celle d'une sphère. Sur l'axe, au point le plus bas la différence de niveau est égale à $0,45 \mathrm{~mm}$ et à $0,25 \mathrm{~mm}$ au point le plus haut. La déformation maximale est donc inférieure à $4 \%$ de $a$. Le centre de gravité du domaine liquide est situé $0,1 \mathrm{~mm}$ plus bas que celui de la partie gauche. Les puissances dissipées par effet Joule sont respectivement de $431 \mathrm{~W}$ pour la sphère et de $421 \mathrm{~W}$ pour la charge déformée, soit une différence de $2 \%$.

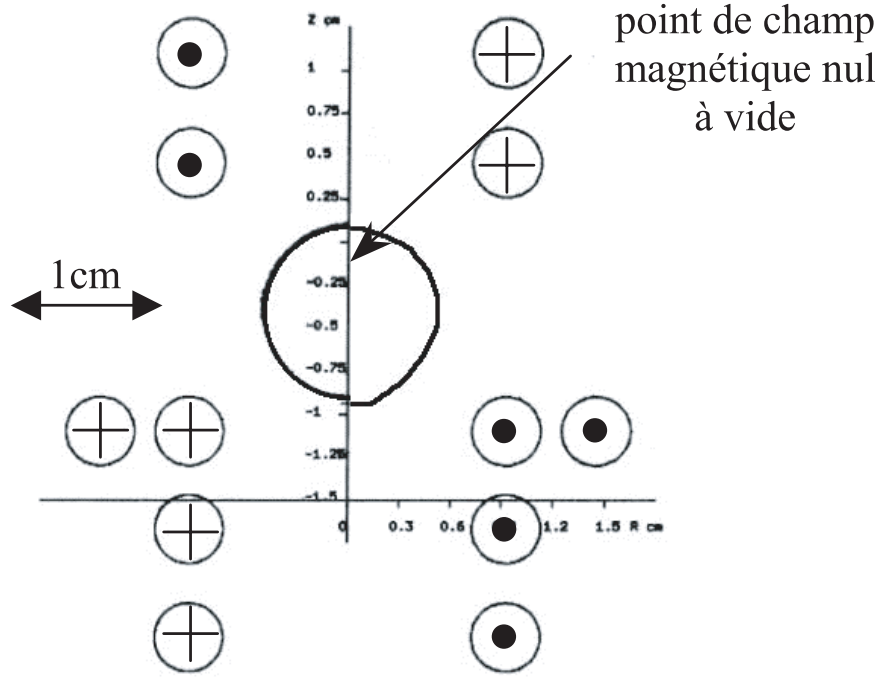

Fig. 7. Calcul de la surface libre dans la configuration Maglev axisymétrique, calcul Ophélie, $I_{\mathrm{eff}}=400 \mathrm{~A}, f=303 \mathrm{kHz}$, rayon de la sphère initiale $a=5 \mathrm{~mm}, \sigma=1,176 \times 10^{6}(\Omega . \mathrm{m})^{-1}$, $\rho=7,9 \times 10^{3} \mathrm{~kg} . \mathrm{m}^{-3}$, à gauche surface sphérique, à droite surface déformée.

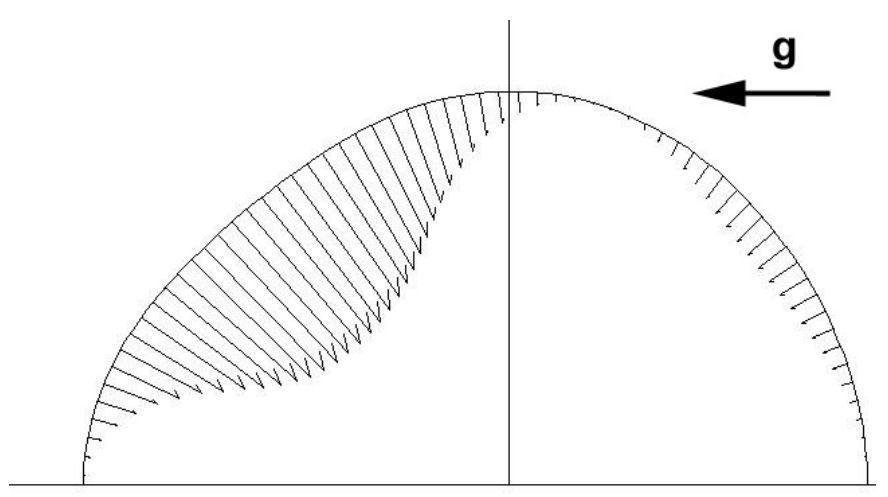

Fig. 8. Forces électromagnétiques sur la surface de la goutte déformée, calcul Induct2D, mêmes conditions que figure 7 .

Afin de calculer correctement l'électromagnétisme, 17 mailles décrivent la peau électromagnétique. Calculées par le module Induct 2D, dans un plan méridien, les forces électromagnétiques à la surface du domaine sont portées sur la figure 8. Conformément à ce qui est attendu, ces forces sont quasiment normales à la surface libre. Elles ont une intensité la plus élevée au voisinage des inducteurs et s'annulent, sur l'axe. En effet, au point bas, seule la tension de surface balance la gravité. Un autre point de cette surface présente aussi une force nulle. Ceci est dû à la présence des deux contre-spires inductives supérieures. Ces forces sont donc fortement rotationelles et engendrent un champ de vitesses à l'intérieur de la goutte.

Le champ de vitesse calculé, pour un frottement nul à la surface, (i.e. $\partial \mathbf{u} /\left.\partial n\right|_{\text {surface }}=0$ ) est présenté sur la figure 9 , pour une hypothèse d'écoulement laminaire. Cette hypothèse est à la limite de sa justification étant donnée la valeur du nombre Reynolds attaché à la vitesse 


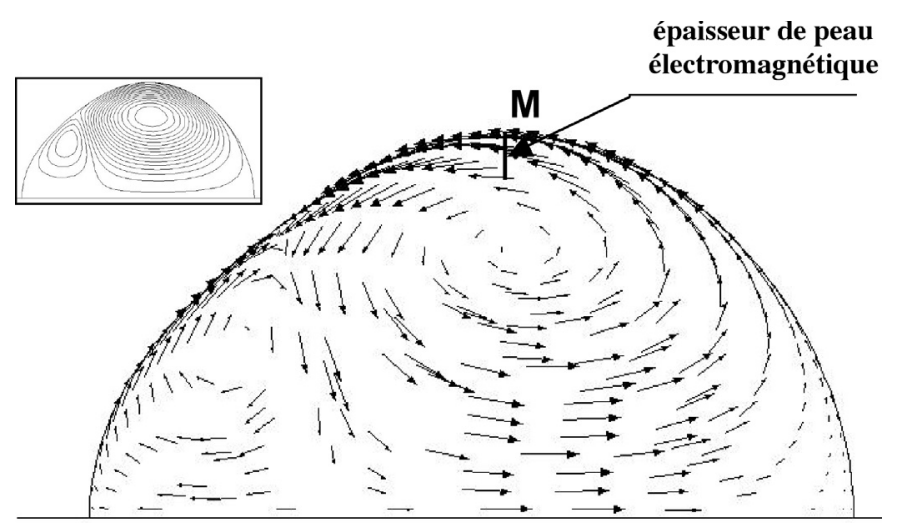

Fig. 9. Champ de vitesse à l'intérieur de la goutte, calcul Fluent, écoulement supposé laminaire, mêmes conditions que figure 7 , dans le cadre en haut à gauche fonction de courant.

d'Alfvén. La bonne convergence de ce calcul laminaire est donc surprenante. On remarque que l'écoulement calculé s'organise en 2 vortex de taille et d'intensité inégale. Les fonctions de courant correspondantes sont portées dans un cadre en haut à gauche de la figure 9. La vitesse calculée maximale est de $1 \mathrm{~m} . \mathrm{s}^{-1}$. La vitesse moyenne est calculée comme l'intégrale de la valeur absolue de la vitesse sur l'axe et est égale à 0,446 m.s ${ }^{-1}$. La vitesse d'Alfvén correspondant au courant inducteur $\left(I=400 A_{\text {eff }}\right)$ est de 0,7 m.s ${ }^{-1}$, l'ordre de grandeur est donc bien respecté.

Le nombre de Reynolds de goutte est égal à 3400. Or, dans un article publié récemment, pour une configuration légèrement différente, Shatrov [11] propose un nombre de Reynolds critique de 100. Ce critère de déstabilisation conduit à penser qu'une turbulence faible existe dans la goutte et qu'un effort de sa description doit être entrepris.

Le profil de la vitesse tangentielle à la surface libre au point $\mathrm{M}$, point de tangente verticale à la surface libre, a été tracé sur la figure 10. Partant de la surface libre, on y remarque un profil constant, conforme à la condition à la limite choisie. Cette vitesse diminue de moitié dans l'épaisseur de peau, et s'annule à 1,41 mm de la surface c'est-à-dire à 2 épaisseurs de peau. On remarque que le choix de la condition de frottement nulle à la surface libre conditionne le résultat du calcul. Le frottement réel est très difficilement quantifiable en l'état de notre connaissance.

\subsection{Mise en place des modélisations numériques avancées}

Notre effort futur portera sur 3 les points :

- la description de l'électromagnétisme notamment des effets 3D responsables d'instabilités de rotation des échantillons, et d'inhomogénéité du chauffage,

- la modélisation des phénomènes transitoires notamment de la description de l'évolution temporelle de la surface libre,

- la modélisation de la faible turbulence qui existe dans ce bain et qui modifie ses propriétés de transport.

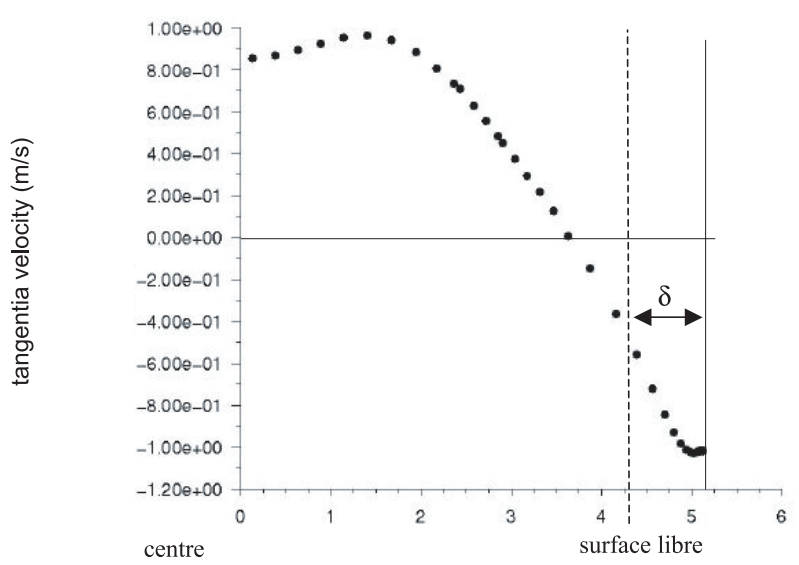

Fig. 10. Profil de la vitesse tangentielle à la surface libre au point $\mathrm{M}$ de normale horizontale mêmes conditions de calcul que figure 9 .

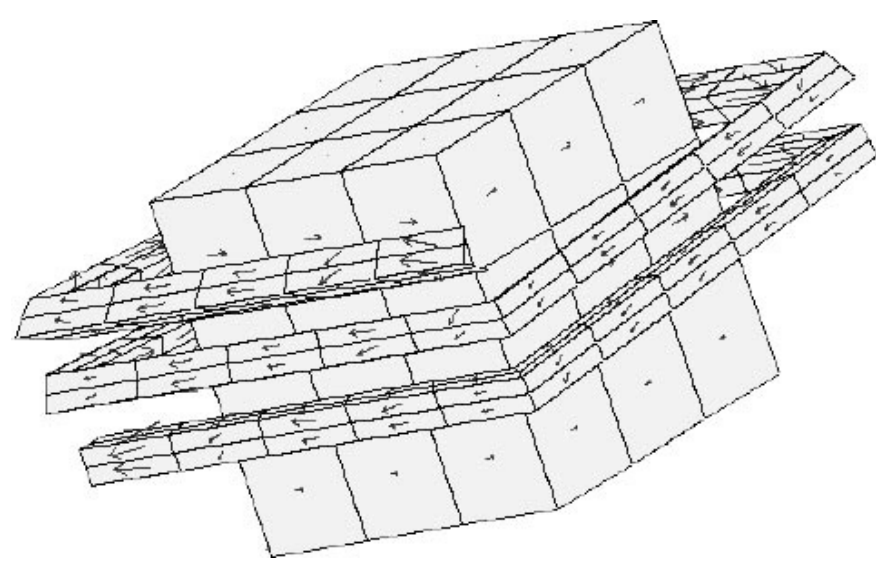

Fig. 11. Répartition des densités de courant à la surface d'un inducteur présentant une hélicité et une charge de section carrée (fréquence : $10 \mathrm{kHz}$ ) calculée à l'aide d'une modélisation numérique 3D basée sur une méthode intégrale.

Une modélisation de la lévitation en conditions non axisymétriques c'est-à-dire $3 \mathrm{D}$, qui permettra la prise en compte de l'hélicité de l'inducteur, des effets de dissymétrie ou de décentrage de la charge sur l'impédance du circuit électrique, ... en relation avec la partie expérimentale a commencé. Un exemple de calcul est donné sur la figure 11 pour un lingot carré entouré par un inducteur présentant une hélicité. Les flèches représentent la densité de courant électrique au centre de chaque facette. On remarque que le code autorise des courants électriques en hélice dans la charge et décrit des effets de bords.

Une des mesures susceptibles d'être réalisée dans Tempus est la mesure de capacité calorifique. Pour cela les courants inducteurs sont modulés et la température à la surface de la charge enregistrée. Pour modéliser la 

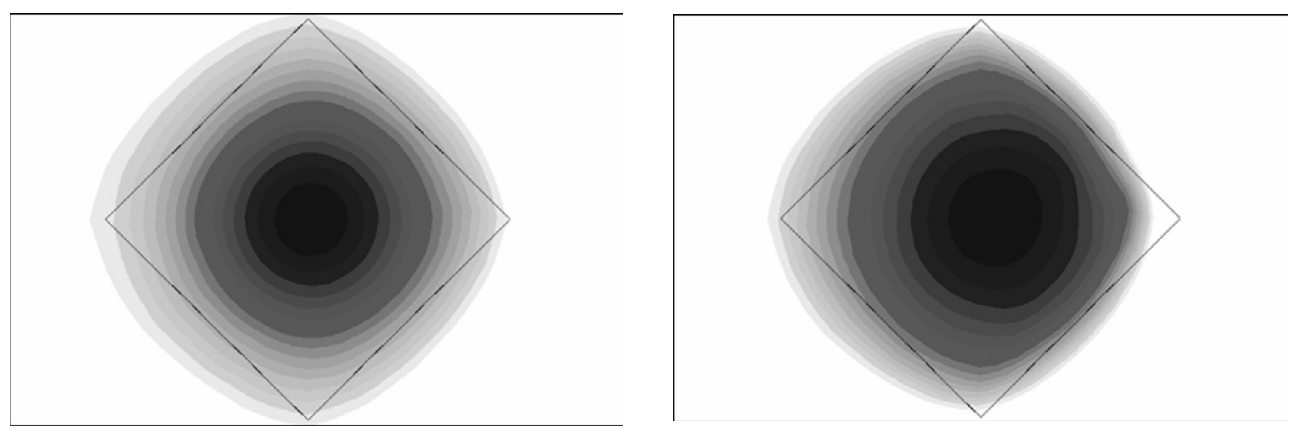

Fig. 12. Exemple de suivi de front au cours du temps par le logiciel FLUX-EXPERT. À l'instant $t=0 \mathrm{~s}$ le barreau d'acier de section carrée est plongé dans un bain d'acier légèrement surchauffé. À gauche l'état du barreau au bout de $1 \mathrm{~s}$; à droite au bout de $2,3 \mathrm{~s}$.

température en surface de la charge au cours du temps, dans un premier temps, l'écoulement dans la goutte sera considéré comme connu. La position instantanée de la goutte sera obtenue par un modèle spécifique implémenté dans le logiciel FLUX-EXPERT. Ce modèle permet de suivre une surface qui se déplace au cours du temps. Un exemple d'application de ce modèle est représenté sur la figure 12 pour un barreau de section carrée plongé dans un bain d'acier légèrement surchauffé. La section du barreau solide croit au cours du temps. À cause des mouvements convectifs dans le bain, la déformation de la surface du barreau n'est pas isotrope, ce qui est bien représenté par le calcul.

\section{Conclusions}

Nous avons présenté :

- l'expérience Tempus et son utilisation pour mesurer des propriétés thermophysiques,

- la contribution de l'équipe du laboratoire EPMMadylam à la modélisation physique et numérique de cette expérience.

L'installation expérimentale de lévitation a fonctionné mais sans son système de mesure. Ce dernier a été essayé sur une autre installation et a montré la faisabilité du système proposé.

La simulation numérique des expériences donne les ordres de grandeur attendus, mais ne peut être considérée comme dimensionnante. Des efforts de description de la turbulence à l'intérieur de la goutte ainsi que du frottement à la surface libre doivent être menés à bien. La description de l'électromagnétisme 3D est en place et doit être appliquée à la géométrie de notre expérience. Le suivi non stationnaire de la surface libre clé de la modélisation de l'évolution de la température de surface au cours du temps a aussi commencé.

\section{Références}

[1] E.C. Okress, D.M. Wroughton, C. Comenetz, P.N. Brace, J.C.K. Kelly, Electromagnetic levitation of solid and molten metals, J. Appl. Phys. 23 (1952) 545

[2] D.L. Cummins, D.A. Blackburn, Oscillations of magnetically levitated aspherical droplet, J. Fluid Mechanics, 224 (1991) 395-416

[3] H. Lamb, Hydrodynamics, Cambridge University Press (1975)

[4] I. Egry, Properties, nucleation and growth of undercooled liquid metals, J. Jpn. Soc. Microgravity Appl., 15(4) (1998) 215-224

[5] V. Bojarevitch, K. Pericleous, Modelling electromagnetically levitated liquid droplet ISIJ International 43(6) (2003) 890-898

[6] Smithells, 2002 Données thermophysiques des métaux, $7^{\text {e }}$ édition, E.A. Brandes, G.B. Brook (edn.)

[7] D. Perrier, Y. Fautrelle, J. Etay, Experimental and Theoretical Studies of the Motion Generated by a TwoFrequency Magnetic Field at the Free Surface of a Gallium Pool Metallurgical \& Materials Transactions 34B(5) (2003) 669-678

[8] D. Perrier, J.P. Paulin, B. Bardet, R. Gerner, Y. Fautrelle, J. Etay, A new way of diagnostic of the state of the load in an induction system, Proceedings of the 4th International Conference on Electromagnetic Processing of Materials, EPM2003, Lyon (France), 14-17 October 2003 (actes sur CD)

[9] A. Gagnoud, I. Leclercq, Electromagnetic modelling of induction melting device in cold crucible, IEEE Transactions on Magnetics 24(1) (1988)

[10] Y. Delannoy, D. Pelletier, J. Etay, Y. Fautrelle, Stirring and mass transfer in a 1-phase induction system, actes de la Conférence Internationale PAMIR Ramatuelle (France), section II (2002) 39-44

[11] V. Shatrov, J. Priede, G. Gerbeth, Three-dimensional linear analysis of the flow in a liquid spherical droplet driven by an alternating magnetic field, Physics of Fluids, 15(5) (2003) 